\title{
Impact of Acute Phase Epigallocatechin-3-gallate Supplementation on Consciousness and S100B Serum Levels in TBI Patients: A Double Blind Randomized Clinical Trial
}

Leila Zareian ${ }^{1}$, Amir Azarhomayoun ${ }^{2}$, Maysam Alimohamadi ${ }^{3}$, Mohammadreza Khajavi ${ }^{4}$, Soodeh Razeghi-Jahromi ${ }^{5^{*}}$

\author{
' MSc, Animal Physiology Department, Basic Sciences Faculty, Shahrekord Branch, Islamic Azad University, Shahrekord, Iran \\ ${ }^{2}$ MD, Sina Trauma and Surgery Research Center, Tehran University of Medical Sciences (TUMS), Tehran, Iran \\ ${ }^{3} \mathrm{MD}$, Brain and Spinal Cord Injury Research Center (BASIR), Neuroscience Institute, Tehran University of Medical Sciences \\ (TUMS), Tehran, Iran \\ ${ }^{4}$ MD, Department of Anesthesiology, Sina Hospital, Tehran University of Medical Sciences, Tehran, Iran \\ ${ }^{5} \mathrm{PhD}$, Department of Clinical Nutrition and Dietetics, Faculty of Nutrition and Food Technology, Shahid Beheshti University of \\ Medical Sciences, Tehran, Iran
}

*Corresponding Author Address: Department of Clinical Nutrition and Dietetics, Faculty of Nutrition and Food Technology, Hafezi Ave., Farahzadi Blv., Tehran, Iran. Telephone: 0098-912-6229144. E-mail:razeghi@sina.tums.ac.ir

Article Type: Clinical Trial

Received: May 6, 2017, Last Revised: May 31, 2017, Accepted: May 29, 2017, Published: September 28, 2017

\section{Abstract}

Background and Aim: Traumatic brain injury is one of the leading causes of mortality and disability in young adults. Epigallocatechin-3-gallate, the antioxidant compound of green tea, has been proposed to have antioxidant and anti-inflammatory properties. This study evaluates the potential effects of epigallocatechin-3-gallate on the early clinical outcome and serum S100B levels (biomarker for brain tissue damage severity) in patients with moderate to severe traumatic brain injury.

Methods and Materials/Patients: Thirty patients with moderate to severe traumatic brain injury admitted to the intensive care unit were enrolled. The patients were randomly allocated to treatment with either a daily oral dose of $400 \mathrm{mg}$ epigallocatechin-3-gallate or placebo (distilled water) for seven days. The main outcome measures were duration of mechanical ventilation and ICU stay, Glasgow Coma Scale, and S100B protein level.

Results: The results revealed a significant improvement in consciousness level after seven days in the epigallocatechin-3-gallate group (2.93 \pm 3.9 unit improvement in GCS versus $0.14 \pm 3.05$ reduction in GCS, p-value:0.033). There was also a significantly shorter duration of mechanical ventilation in the epigallocatechin-3-gallate compared to the control group (5.1 days versus 9.8 days, p-value:0.02). Reduction of the serum S100B level was slightly higher in the epigallocatechin-3-gallate group (23.96 versus $18.6 \mathrm{pg} / \mathrm{ml}$ ) but the difference was not statistically significant.

Conclusion: Epigallocatechin-3-gallate supplementation had beneficial effects on consciousness level of the patients with moderate to severe traumatic brain injury in the acute phase.

Keywords: Epigallocatechin-3-gallate; Head Trauma; Neuroprotection; S100B Protein; Traumatic Brain Injury

Please cite this paper as: Zareian L, Azarhomayoun A, Alimohamadi M, Khajavi M, Razeghi-Jahromi S. Impact of
Acute Phase Epigallocatechin-3-gallate Supplementation on Consciousness and S100B Serum Levels of TBI Patients: A
Double Blind Randomized Clinical Trial. Iran J Neurosurg. 2017;3(2):51-57

Introduction

Traumatic brain injury (TBI) is the leading cause of disability and death in all age groups worldwide [1]. TBI occurs as a result of direct mechanical insult to the brain followed by secondary events resulting in cell degeneration and death. Secondary insults include excitotoxic damage, blood brain barrier disruption, free radical production, calcium-mediated damage, neuro-inflammation, and hypoxia due to circulatory disturbance [2-4]. Since the primary insult is not preventable, investigations are mainly focused on reversing the secondary insults $[5,6]$. Recent studies have shown that oxidative stress has a central role in the pathogenesis 
of traumatic brain damage $[5,6]$. Oxidative stress enhances free radical generation that subsequently activates apoptotic pathways which results in cell death. Neurodegeneration might continue for months after TBI.

Green tea polyphenol, epigallocatechin3-gallate (EGCG), has a variety of biological functions and health benefits including antiobesity [7], anti-inflammatory [8], antiatherogenic [9] and neuro-protective [10] properties.

EGCG is also known as a very potent antioxidant in biological systems. Several mechanisms have been proposed for antioxidant effects of EGCG including free radical scavenging, attenuation of lipid peroxidation by inhibiting xanthine oxidase activation and blockade of inducible nitric oxide synthase [11-13].

Recent in vivo and in vitro studies suggest the protective role of EGCG against free radical-induced neuronal damage $[14,15]$. Studies have indicated that S100B, a member of the $\mathrm{S} 100$ protein family, is abundant in the nervous system where it is predominantly expressed in astrocytes, oligodendrocytes, and Schwann cells. When secreted by astrocytes, S100B has neurotrophic effects during development and nerve regeneration at physiologic concentrations. However, high concentrations of S100B have shown to be neurotoxic. It seems that $\mathrm{S} 100 \mathrm{~B}$ serum levels correlates with clinical outcome after TBI [16]. Some studies suggested S100B as a biomarker for determining the severity of brain injury and also monitoring therapeutic intervention [17]. An increment in S100B level was reported immediately after TBI. The level was reported to drop after treatment. There is no evidence about the impact of administration of EGCG on the outcome of the patients with TBI in the acute phase. This study is a randomized clinical trial to evaluate the potential benefit of EGCG on the early clinical outcome and serum levels of S100B biomarker of the patients suffering from moderate to severe TBI.

\section{Methods and Materials/Patients}

Male patients (16-65 years old) admitted to a referral trauma center, intensive care unit of Sina hosipital in Tehran, Iran, with moderate to severe TBI from October 2014 to October 2015 were enrolled in this study. The inclusion criteria of this study were GCS of 4-12, enteral nutrition starting within 24 hours after admission and mechanical ventilation. The procedure and protocol of the study was approved by the Ethic Committee of Tehran University of Medical Sciences (clinical trials.gov ID: NCT02731495). Written informed consent was obtained from patients' relatives.

\section{Data Collection and Supplementation}

After recording anamnesis, the patients were examined physically. Emergency care started in the emergency room. Patients with GCS higher than 12 or lower than 4, internal organ bleeding, obvious fractures in their limbs, history of underlying neurologic, metabolic, or psychiatric disorder, alcohol or drug abuse, and vegetarian diet were excluded from the study. Basic information including age, sex, vital signs, GCS, and clinical symptoms at the time of admission were recorded.

All patients received enteral nutrition with the same formula and protocol. The patients were divided into two groups using random balanced blocks: (1) receiving EGCG supplement $(\mathrm{n}=15)$ and $(2)$ receiving placebo (control group, $n=15$ ). CONSORT flow diagram of the study is shown in figure 1. EGCG supplement was in form of 400 mg oral capsules with the purity of $80 \%$ catechin (Vitamilife, U.S.). EGCG powder of each capsule was dissolved in $10 \mathrm{ml}$ deionized water and given to patients via gavage (one capsule per day) for seven days by a nurse who was blinded to the treatment. The control group received only $10 \mathrm{ml}$ of deionized water via gavage for a week by the same nurse. During that week, GCS of the patients was recorded by a neurosurgery resident blinded to the intervention. Vital signs and serum level of glucose, hematocrit/hemoglobin, and platelets were recorded every day at 9:00 AM for one week. Moreover, the durations of mechanical ventilation and ICU stay were recorded.

In order to analyze $\mathrm{S} 100 \mathrm{~B}$ protein, a $5 \mathrm{ml}$ peripheral venous blood sample was taken from patients on admission (T1) and 24 hours after the last gavage with EGCG (T2) 


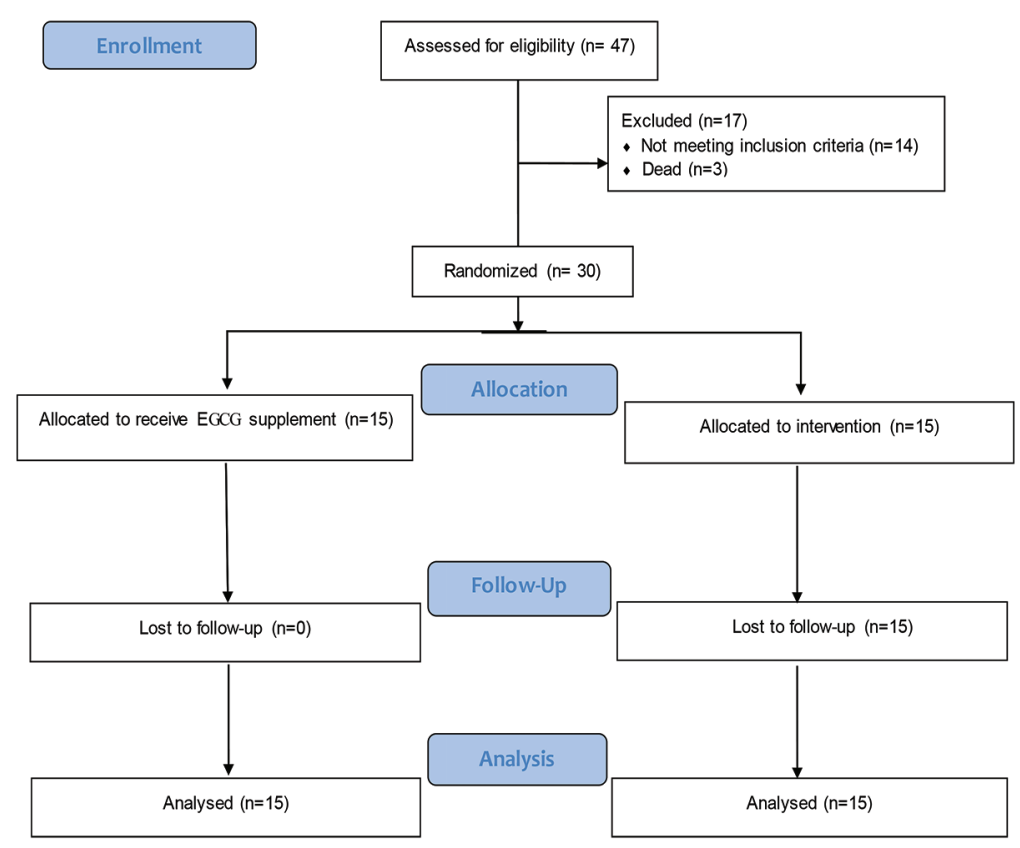

Figure 1. CONSORT Flow Diagram of the Study

and stored at $-80^{\circ} \mathrm{C}$. Human S100B ELISA kit (BioVendor - Laboratorni medicina a.s. Cat\# RD 192090100) was used to quantify the level of S100B protein according to the manufacturer protocol. The primary outcome in this study was serum level of the S100B biomarker on admission and after one week of supplementation with either placebo or EGCG.

\section{Statistical Analysis}

The distribution of continuous variables was assessed using Kolmogorov-Smirnov test. To compare the continuous variables between the two groups, the independent -samples t-test and Mann-Whitney U-test were used for data with normal and nonnormal distributions, respectively. For a before-after comparison of the variable levels, paired sample t-test and Wilcoxon were used for the data with normal and nonnormal distribution, respectively. P-value less than 0.05 was considered as statistically significant for all tests. The data were analyzed using SPSS version 21 (SPSS Inc., Chicago, IL, USA).

\section{Results}

The baseline characteristics of the patients are presented in table 1. The two groups had no significant differences in baseline characteristics. As shown in table 2, seven days following TBI, the last GCS was significantly higher in the EGCG group. Alteration of GCS was measured as last GCS on ICU admission. The EGCG group had significantly higher GCS improvement than the control group after seven days: the mean GCS level increased by 2.93 units in the EGCG group while having an average of 0.14-unit deterioration in the control group at the same time.

Alteration of S100B level was calculated as T2-T1. The average S100 levels decreased in both groups after seven days of admission. The amount of reduction in S100B levels was greater in EGCG than that in the control group (23.96 pg/ml versus $18.6 \mathrm{pg}$ / $\mathrm{ml})$ but the difference was not statistically significant $(\mathrm{P}=0.209)$.

Although the overall duration of ICU stay was not significantly different among the two groups, the duration of mechanical ventilation was significantly shorter in the EGCG group (5.1 versus 9.8 days) (Table 3).

The patients were divided into those who did not have any clinically significant abnormality of the serum gluocose, $\mathrm{Hb}$ / 
Table 1. Clinical Characteristics of the Study Groups

\begin{tabular}{|c|c|c|c|}
\hline Patient Characteristics & EGCG & Placebo & P.Value \\
\hline Age (year) & $45.67 \pm 17.7 \S$ & $47.93 \pm 16.3 \S$ & $0.381 \dagger$ \\
\hline GCS on Admission & $6.26 \pm 2.4$ & $6.9 \pm 2.8$ & $0.028 \dagger$ \\
\hline Cause of Injury & & & $0.283 ¥$ \\
\hline Car or Motor Accident & $12(80 \%)$ & $14(93.3 \%)$ & $0.283 ¥$ \\
\hline Falling & $3(20 \%)$ & $1(6.7 \%)$ & \\
\hline CT Abnormalities and Other Injury & & & $0.624 ¥$ \\
\hline Characteristics & $2(13.3 \%)$ & $3(20 \%)$ & $0.624 ¥$ \\
\hline Cortical Contusion & $2(13.3 \%)$ & $3(20 \%)$ & $0.543 ¥$ \\
\hline Subdural Hematoma Evacuate & $2(13.3 \%)$ & $1(6.7 \%)$ & $0.543 ¥$ \\
\hline Subdural Hematoma & $1(6.7 \%)$ & $2(13.3 \%)$ & $0.721 ¥$ \\
\hline Epidural Hematoma & $3(20 \%)$ & $3(20 \%)$ & $0.543 ¥$ \\
\hline Intracerebral Hematoma & $2(13.3 \%)$ & $1(6.7 \%)$ & $0.624 ¥$ \\
\hline Depressed Skull Fracture & $3(20 \%)$ & $2(13.3 \%)$ & $0.639 ¥$ \\
\hline Diffuse Axonal Injury & $2(13.3 \%)$ & $2(13.3 \%)$ & \\
\hline Patient Mortality during the Study & & & \\
\hline
\end{tabular}

Data Are Presented as Number (Percent), Otherwise Stated. § Mean \pm SD, $\uparrow$ Independent Samples T test, ¥ Pearson Chi-square

Table 2. Comparison of the Main Outcome Measures among the Two Study Groups

\begin{tabular}{|l|c|c|c|}
\hline \multirow{2}{*}{\multicolumn{1}{|c|}{ Outcome Variable }} & \multicolumn{2}{c|}{ Study Group } & \multirow{2}{*}{ P-value } \\
\cline { 2 - 4 } & EGCG Group & Placebo Group & $0.013^{*}$ \\
\hline Last GCS & $10.5 \pm 2.6$ & $7.6 \pm 3.3$ & $0.797^{*}$ \\
\hline Last S100B Level $(\mathbf{p g} / \mathbf{m l})$ & $30(20-120)$ & $89(40-540)$ & $0.033^{*}$ \\
\hline Alteration of GCS & $2.93 \pm 3.9$ & $0.14 \pm 3.05$ & $0.209^{*}$ \\
\hline Alteration of S100B Level $(\mathbf{p g} / \mathbf{m l})$ & $23.96 \pm 41.9$ & $18.6 \pm 53.78$ & \\
\hline
\end{tabular}

Data Are Presented as Mean \pm SD, Otherwise stated. * Paired Samples T test, £Wilcoxon Signed-Ranks Test, ๆ Median (Minimum-Maximum), $\dagger$ Independent Samples T test.

Table 3. Comparison of the Duration of ICU Stay, Mechanical Ventilation Period and Laboratory Findings among the Study Groups

\begin{tabular}{|c|c|c|c|c|}
\hline \multirow{2}{*}{\multicolumn{2}{|c|}{ Outcome Variable }} & \multicolumn{2}{|c|}{ Patient Group } & \multirow{2}{*}{ P-value } \\
\hline & & EGCG Group & Placebo Group & \\
\hline \multicolumn{2}{|l|}{ Duration of ICU Stay (days) } & $14.27 \pm 8.2 \S$ & $15.60 \pm 6.5 \S$ & $0.627 \dagger$ \\
\hline \multicolumn{2}{|c|}{ Duration of Mechanical Ventilation (days) } & $5.1(2-17)$ & $9.8(4-19)$ & $0.02 \ddagger$ \\
\hline \multirow{3}{*}{$\begin{array}{c}\text { Number of Patients with } \\
\text { Clinically Significant } \\
\text { Abnormalities }\end{array}$} & Serum Glucose & $2(13.3 \%)$ & $3(20 \%)$ & $0.36 \ddagger$ \\
\hline & $\mathrm{Hb} / \mathrm{Het}$ & $2(13.3 \%)$ & $2(13.3 \%)$ & $0.91 \ddagger$ \\
\hline & Platelet & $1(6.6 \%)$ & 0 & $0.42 \ddagger$ \\
\hline
\end{tabular}

Data Are Presented as Mean \pm SD, Otherwise Stated. $€$ Paired Samples T test, £Wilcoxon Signed-Ranks Test, $q$ Median (Minimum-Maximum), $\dagger$ Independent Samples t Test. 
Hct and platelet levels (not requiring therapeutic intervention) and those who required therapeutic intervention for abnormalities of these parameters. The prevalence of these potentially confounding factors was not statistically significant between the two groups of the study (Table3).

\section{Discussion}

In this study, we evaluated the effect of EGCG of green tea on the clinical outcome (consciousness, duration of ICU stay, mechanical ventilation, and serum S100B levels) of the patients with moderate to severe TBI. Our results show a beneficial effect of EGCG supplementation on improvement of GCS and shortening of the mechanical ventilation period in these patients. These results are in consistence with the previous experimental studies: Itoh et al. reported that seven days of oral supplementation with $0.1 \%$ solution of EGCG in water for brain injury-induced rats could reduce neuronal damages [18]. Hau et al. reported that intraperitoneal injection of epigallocatechin-3-gallate solution has neuroprotective effects in brain damage-induced rats by protecting them against oxidative damages to the brain. They concluded that EGCG showed neuroprotective effects by reducing free radical damages and preventing apoptosis in damaged neurons $[19,20]$.

A number of reports proposed S100B serum level as a valid biomarker for brain damage [21]. Previous studies showed that the injection of five mg S100B protein to rat cerebellar vermis had anti-apoptotic effects [22]. Some studies show that S100B probably binds to the neuronal membrane RAGE receptors in low concentrations and increases the survival of neurons by producing against reactive oxygen species (ROS) and increasing the expression of anti-apoptotic factors [22-25]. The findings of current study show that EGCG supplementation results in reducing S100B protein compared with the control group, although the difference between the two groups was not statistically significant.

Pro-inflammatory cytokines such as tumor necrosis factor alpha (TNF- $\alpha)$ have an important role in the pathophysiology of cerebral edema and brain damage after traumatic insult [26,27]. Factors with anti-inflammatory properties can increase the blood flow in damaged tissues, and subsequently improve the process of wound healing and recovery [27]. Experimental evidence suggests that EGCG could inhibit TNF-alpha gene expression $[28,29]$. In a study performed on an animal model of multiple sclerosis, the combination of glatiramer acetate (GA) and EGCG had favorable effects on neuronal survival and axonal growth by reduced reactive oxygen species generation and reduction in the nuclear factor kappa-light-chain-enhancer of activated B cells (NFkB) [28]. To the best of our knowledge, until the date no human study has assessed the effect of EGCG supplementation on the secondary neural damages caused by trauma [30].

To control the confounding effect of underlying mental illness and/or internal organ injury on the clinical outcome of TBI, we excluded these patients from our study. The other possible source of confounding was hematologic and metabolic derangements which may affect the clinical outcome in TBI patients. Statistical analysis revealed that prevalence of these parameters was not statistically different among the two groups, and thus may not serve as a source of confounding. The major limitation of this study was the small sample size of the study. Due to the overwhelming predominance of TBI among men in our country, we only included male patients in this study. Furthermore, impact of the findings of this study on the long-term clinical outcome of TBI patients is a very important issue that needs to be evaluated by larger studies with longer follow-up periods.

\section{Conclusion}

The results of this study show that acute phase supplementing with EGCG might have favorable effects on consciousness levels and duration of mechanical ventilation in early phase after moderate to severe TBI. According to the availability and low cost of this product, we recommend early treatment with EGCG as a part of the integral management of patients with TBI admitted to ICU. 


\section{Acknowledgement}

We would like to appreciate the staff of Sina hospital ICU for their kind cooperation. Also, we would like to thank Ms. Hamideh Abbasi for her assistance in assessing S100B level.

\section{Funding}

This study was supported by a grant from Iranian Center of Neurological Research, Tehran University of Medical Sciences.

\section{Conflicts of Interest}

The authors declare that they have no conflict of interest.

\section{Authors' Contribution}

Conception and Design: Leila Zareian, Soodeh Razeghi Jahromi*

Data Collection: Leila Zareian, Amir Azarhomayoun

Drafting the Article: Leila Zareian, Amir Azarhomayoun, Mohammadreza Khajavi

Critically Revising the Article: Maysam Alimohamadi

Reviewed Submitted Version of the Manuscript: All Authors

Approved the Final Version of the Manuscript: All Authors

\section{References}

1. Bruns JR, Hauser WA. The epidemiology of traumatic brain injury. a review. Epilepsia. 2003; 44: 2-10.

2. Werner C, Engelhard K. Pathophysiology of traumatic brain injury. Br J Anaesth. 2007;99: 4-9.

3. Leon-Carion j, Barroso Y marrtin JM. Epidemiology of traumatic brain injury and subarachnoid hemorrhage. $J$ Pituitary. 2005; 8: 197-202.

4. Faria A, Meireles M, Fernandes I, Santos-Buelga C, Gonzalez-Manzano S, Dueñas M, de Freitas V, Mateus N, Calhau C. Flavonoid metabolites transport across a human BBB model. Food Chem. 2014 Apr 15;149:190-6.

5. Brain Trauma Foundation; American Association of Neurological Surgeons; Congress of Neurological Surgeons; Joint Section on Neurotrauma and Critical Care, AANS/CNS, Bratton SL, Chestnut RM, Ghajar J, McConnell Hammond FF, Harris OA, Hartl R, Manley GT, Nemecek A, Newell DW, Rosenthal G, Schouten J, Shutter L, Timmons SD, Ullman JS, Videtta W, Wilberger JE, Wright DW. Guidelines for the management of severe traumatic brain injury. I. Blood pressure and oxygenation. J Neurotrauma. 2007;24 Suppl 1:S7-13

6. Alexander M. Mild traumatic brain injury pathophysiology, natural history, and clinical management. Neurology. 1995; 45(7): 50 - 60 .

7. Kuptniratsaikul V, Kovindha A, Suethanapornka S.Complications during the rehabilitation period in thai patients with stroke: A Multi center prospective study AMJ Phys Med Rehabil. 2009; 88(2): 92-99.

8.Castellano C. Psychopharmacology of memory modulation: evidence hormones.J Behave Brain Res. 1996; 34(8): $1-21$.

9. Rosenfeldt F, Wilson M. population, pathophysiology and therapy. J Exp Gerontol. 2013;48:45-54.
10. Chen SD, Yang DI, Lin TK, Shaw FZ, Liou CW, Chuang YC. Roles of oxidative stress, apoptosis, PGC-1 and mitochondrial biogenesis in cerebral ischemia. J Mol Sci . 2011; 12: 7199-215.

11- Higdon J. Tea catechins and polyphenols: health effects, metabolism, and antioxidant functions.J Food Sci Nutr. 2003;43: 89-143.

12. Anna K. Flavonoids and the CNS, Molecules. 2011; 16: 1471-1485.

13. Croft KD. (1998). The chemistry and biological effects of flavonoids and phenolic acids. Ann NY Acad Sci. 854:435.

14. Middleton E J. Effect of plant flavonoids on immune and inflammatory cell function. Adv Exp Med Biol. 2000; 439:175-82.

15. Hanasaki Y, Ogawa S, Fukui S. The correlation between active oxygens scavenging, and antioxidative effects of flavonoids. Free Radic Biol Med. 1994;16:845-50

16. BloomfieldSM, Mcknney J, Smith L, Brisman. Reliability of S100B in predicting severity of central nervous system injury. Neurocritic Care. 2007; 6:121-138. 17. Woertgen C, Holzschuh M, Metz C, Brawanski A. Comparison of clinical, radiologic, and serum marker as prognostic factors after severe head injury. J Trauma. 1999; 47: 1126-1130.

18. Itoh T, Tabuchi M, Mizuguchi N, Imano M, Tsubaki M, Nishida S. Neuroprotective effect of epigallocatechin3 -gallate in rats when administered pre- or post-traumatic brain injury. J Neural Transm. $2013 ; 120(5): 767-783$.

19. Hua W, Hui-Chin Tu, and Jeng-Yung.Epigallocatechin gallate attenuates $\mathrm{NADPH}-\mathrm{d} / \mathrm{nNOS}$ expression in motor neurons of rats following peripheral nerve injury. J BMC Neuroscience. 2011; 12:52.

20. Zink BJ, SymzdYnjer-Chodobska J, Chodobski A Emerging concepts in the pathophysiology of traumatic brain injury. Psychiat Clin North Am. 2010; 33: 741-756.

21. Karpiak SE, Serokosz M, Rapport MM. Effects of antisera to $\mathrm{S} 100$ protein and to synaptic membrane fraction on maze performance and EEG. Brain Res. 1976; 313- 21.

22. Kleindienst A, McGinn M, Harvey H, Colello RJ, Hamm RJ, Bullock MR. Enhanced hippocampal neurogenesis by intraventricular S100B infusion is associated with improved cognitive recovery after traumatic brain injury. $\mathrm{J}$ Neurotrauma. 2005;22(2):645-655.

23. Hanasaki Y, Ogawa S, Fukui S. The correlation between active oxygens scavenging, and antioxidative effects of flavonoids. Free Radic Biol Med . 1994; 16:845-5.

24. Mirrahimi B, Mortazavi A, Nouri M, Ketabchi E, Amirjamshidi A, Ashouri A, Khajavi M, Mojtahedzadeh M. Effect of magnesium on functional outcome and paraclinical parameters of patients undergoing supratentorial craniotomy for brain tumors: a randomized controlled trial. Acta Neurochir. 2015; 157(6):985.

25. Etezadi F, Aklamli M, Najafi A, Khajavi M, Shariat Moharari R, Mirrahimi B, Mortazavi SA, Mojtahedzadeh M. Evaluation of the anti-inflammatory effects of peri-operative infusion of magnesium sulfate on the microsurgical procedures for intracranial tumors. Anesth Pain Med. 2014;30;4(5):e22379.

26. Chen SD, Yang DI, Lin TK, Shaw FZ, Liou CW, Chuang YC. Roles of oxidative stress, apoptosis, PGC-1 and mitochondrial biogenesis in cerebral ischemia. J Mol Sci. 2011; 12: 7199-215.

27. Chan PH.Reactive oxygen radicals in signaling and damage in the ischemic brain. J Cereb Blood Flow Metab. 2001; 21(1):2-14.

28. Yun HJ, Yoo WH, Han MK, Lee YR, KiLee SI. Epigallocatechin-3-gallate suppresses T NF a lpha -induced $\mathrm{p}$ roduction of M MP-1 a nd -3 i $\mathrm{n} r$ heumatoid arthritis synovial fibroblasts. Rheumatol Int. 2008;29(1): 23-9.

29. Renno WM, Saleh F, Klepacek I, Al-Khaledi G, Ismael $\mathrm{H}$, Asfar S. Green tea pain modulating effect in sciatic nerve chronic constriction injury rat model. Nutr Neurosci. 2006; 
Epigallocatechin-3-gallate (EGCG) and Traumatic Brain Injury

9(1-2): 41-7.

30. Herges K. Neuroprotective Effect of Combination Therapy of Glatiramer Acetate and Epigallocatechin-3-

Gallate in Neuroinflammation. PLoS ONE. 2011; 6(10):

e25456. https://doi.org/10.1371/journal.pone.0025456 